\title{
The VDRL test in a blood transfusion service
}

\author{
R. W. BEAL AND D. J. MERRY
}

From the Red Cross Blood Transfusion Service, South Australian Division, Adelaide, South Australia and the Institute of Medical and Veterinary Science, Adelaide, South Australia

SYNOPSIS A survey has been made of the results of 12 months' VDRL screening in a blood transfusion service. Positive VDRL tests were found on 318 of the 73350 blood donations collected during 1974. Thirty-four tests confirming specific treponemal infection were found in 24 donors. A battery of confirmatory tests showed the remaining 284 positive VDRL reactions from 235 donors to be biological false positive (BFP) results, and, of these, one-third were considered entirely negative by the reference laboratory.

BFP reactions were commoner in female donors than in males, and a higher incidence than expected was observed in younger female donors and older male donors. A marked seasonal incidence was noted, $65 \%$ of all BFP tests from the transfusion service being found in the four colder months of May to August.

Although some differences exist between the group as a whole and the 36 donors (15.3\%) found to have BFP results on more than one occasion during the year, there is little evidence from these studies to suggest that regular blood donation per se is a contributing factor to the finding of a BFP result in VDRL screening. It is probable that the population tested regularly by the blood trans fusion service reflects a small but representative sample of the community as a whole.

Quality control and recipient protection are fundamental operating principles in any blood transfusion service. Because it has been known for more than 100 years that syphilis can be transmitted by the infusion of blood from a patient in the secondary stage of the disease (Keynes, 1949), a serological screening test for syphilis is a time-honoured control procedure in all blood transfusion services. It is not always remembered that such screening tests may occasionally be negative in the primary stages of the disease (Keynes, 1949; Schofield, 1972). The refrigerated storage of anticoagulated blood for 72 hours has reduced to a minimum the risk of inadvertent transmission of syphilis.

Although every blood donation from each blood donor is tested for syphilis, the incidence of confirmed positive tests is low, because the incidence of syphilis within the Australian and other similar communities is relatively low. However; for every confirmed positive subject there is a large number of blood donors who demonstrate a biological false positive reaction (BFP) to one or more of the standard laboratory tests. These donors are of clinical interest since the BFP reaction, if chronic, may

Received for publication 12 May 1975. indicate the presence of an underlying disease process other than treponemal infection. They are also of interest because blood donation per se has been invoked as a cause of a BFP reaction (Garner and Backhouse, 1970; Moore and Mohr, 1952) in VDRL testing.

The more recent advocates of this thesis (Garner and Backhouse, 1970, 1971) have been criticized (Verso, 1971) for failing to look at those blood donors showing BFP reactions for syphilis as part of a 'normal' donor population.

This present study is based on a retrospective analysis of syphilis screening in the Red Cross Blood Transfusion Service (RCBTS) in South Australia during 1974, and examines the age, sex, and seasonal incidence of true positive and BFP reactions to VDRL testing, together with the patterns obtained on subsequent detailed investigation by the reference laboratory.

\section{Materials and Methods}

All blood donations collected in South Australia are screened for the presence of Treponema pallidum infection by the Venereal Disease Research Laboratory (VDRL) test. A clotted specimen of blood is 
obtained from the donor at the completion of the collection of $430 \mathrm{ml}$ of whole blood. The serum from this sample is inactivated by heating at $56^{\circ} \mathrm{C}$ for 30 minutes. Two drops of the inactivated serum are mixed with a drop of dilute cardiolipin antigen, as recommended by the manufacturer (Commonwealth Serum Laboratories), mechanically agitated for four minutes, and read under illuminated magnification ('Maggy lamp').

When a positive result is obtained on VDRL testing, the remainder of the serum is sent to the Serology Department of the Institute of Medical and Veterinary Science (IMVS). In addition to a repeat VDRL test, cardiolipin Wassermann reactions to Whitechapel (WR) and Kolmer techniques (K), a Reiter protein complement fixation test (RPCFT), and a fluorescent treponemal antibody absorption test (FTA-ABS) to the US Department of Health manual (1969) methods, are performed. When a BFP reaction is obtained, the blood donation is not transfused as whole blood but is submitted for fractionation.

\section{Results}

During 1974, 73350 blood donations were collected at the main blood centre in Adelaide and on mobile unit visits to metropolitan and country locations. Of these, $318(0.43 \%)$ were found to be positive to VDRL testing. Confirmatory evidence for syphilis was obtained on 34 of these samples. The incidence of BFP reactions was therefore $0.39 \%$ of all donations tested.

The 34 positive tests for syphilis were found in 24 different donors; the remaining 284 tests regarded as BFPs were found in a total of 235 donors. Twentysix donors were found to have a BFP reaction for syphilis on two occasions during the year, seven donors had BFP reactions on three occasions, and three donors had BFP reactions on four occasions, the maximum number of donations which the Australian blood donor may give during any 12month period.

CONFIRMED POSITIVE TESTS FOR SYPHILIS Twenty-four donors were found to have positive tests for syphilis when fully investigated. Of these, 11 had previous confirmed positive tests prior to 1974, while the remaining 13 had either untreated infection diagnosed for the first time or inactive or effectively treated disease recognized as such for the first time by the Transfusion Service. There were 10 subsequent tests performed on six of these 13 donors during the year.

Sixteen males and eight females were found to have positive tests, and the average ages, 44.6 years for males and 38.0 for females, were somewhat higher than the ages of donors with BFP reactions for syphilis. No seasonal incidence was noted in the occurrence of these confirmed positive reactions.

The serological reaction patterns obtained on initial testing in 1974 for the positive donors are shown in table I. In general terms, the patterns obtained on repeat testing were similar to those on initial testing. The donor showing pattern $F$ had previously been detected as a specific positive, and gave this reaction on two occasions during the year; his serum was routinely sent for detailed investigation, although his VDRL was negative on each occasion at both the RCBTS and at IMVS. Two others, one initially showing pattern $\mathrm{C}$ and the other pattern $\mathrm{D}$, were found by IMVS testing to be positive only for RPCFT and FTA-ABS on subsequent testing.

\section{BIOLOGICAL FALSE POSITIVES}

Sex and Age

Of the 235 blood donors who gave a BFP reaction to VDRL screening during the year, 115 were males and 120 females. This represents an increase over the expected number of female donors, since the RCBTS in South Australia sees between two and three male donors for each of its female donors (Beal, 1965, 1967).

The average age of the 115 males was $37.03 \pm$ 11.96 years and of the 120 females, $32.23 \pm 12.06$ years, both groups being somewhat older than the previously reported (and presently observed) average ages of South Australian blood donors (Beal, 1965, 1967). In order to assess more precisely this apparent

\begin{tabular}{|c|c|c|c|c|c|c|}
\hline Pattern & $V D R L$ & $W R$ & $\boldsymbol{K}$ & $R P C F T$ & $F T A-A B S$ & Number \\
\hline $\begin{array}{l}\mathbf{A} \\
\mathbf{B} \\
\mathbf{C} \\
\mathbf{D} \\
\mathbf{E} \\
\mathbf{F} \\
\mathbf{G} \\
\text { Total }\end{array}$ & $\begin{array}{l}+ \\
+ \\
+ \\
+ \\
+ \\
+ \\
+\end{array}$ & $\begin{array}{l}\overline{-} \\
\overline{+} \\
\overline{+} \\
\overline{+}\end{array}$ & $\begin{array}{l}\overline{+} \\
\overline{-} \\
+ \\
+ \\
\overline{+}\end{array}$ & $\begin{array}{l}- \\
- \\
+ \\
+ \\
+ \\
+\end{array}$ & $\begin{array}{l}+ \\
+ \\
+ \\
+ \\
+ \\
+ \\
+\end{array}$ & $\begin{array}{r}4 \\
2 \\
6 \\
1 \\
6 \\
1 \\
4 \\
24\end{array}$ \\
\hline
\end{tabular}

Table I Confirmed specific infection 


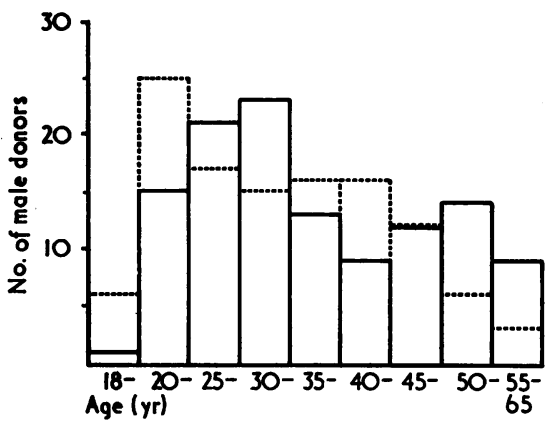

Fig 1 Male donors are shown divided into the age groups specified. The donors having BFP results are shown by the unbroken line, and the broken line represents the previous data (Beal, 1965) for male donors in this Service.

difference, the 235 donors were divided into age groups for purposes of comparison with previously reported data from this Service (Beal, 1965, 1967). It was found (fig 1) that there were more older men with BFP results for VDRL testing than expected, and more younger women (fig 2) than expected with similar results. In both cases, these differences were statistically significant.

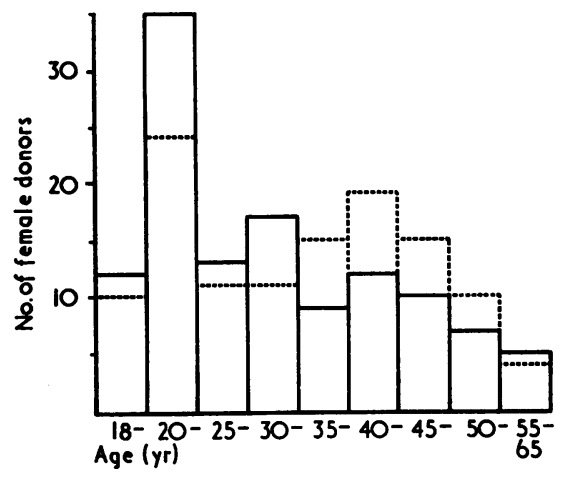

Fig 2 Female donors are shown divided into the age groups specified. The donors having BFP results are shown by the unbroken line, and the broken line represents the previous data (Beal, 1965) for female donors in this Service.

\section{Seasonal Incidence}

When the VDRL BFP results are assessed on the basis of their calendar incidence, a marked increase is noted in the four-month period May to August (fig 3) which covers the late autumn and winter period in the southern hemisphere. The BFP VDRL tests for these four months represent $65 \%$ of all

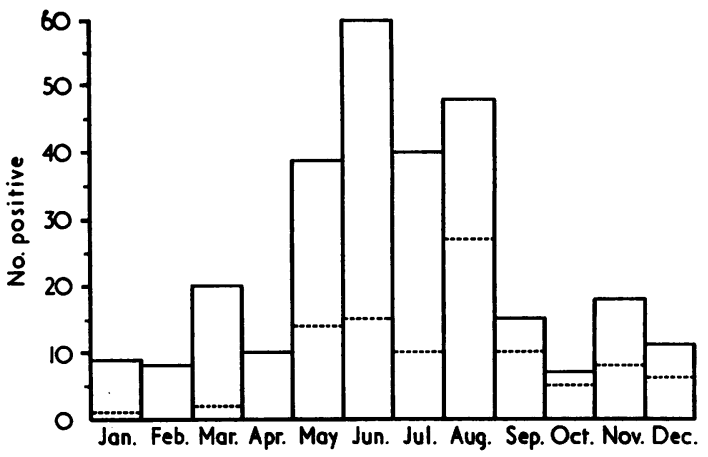

Fig $3 V D R L$ positive results found at the transfusion service shown on a monthly basis. The lower part of the column shows the number that were subsequently found to be negative by the reference laboratory.

BFPs reported for the calendar year. Figure 3 also shows the 99 VDRL tests found positive at the RCBTS which were not confirmed on subsequent testing at the IMVS. While these are discussed below in detail, it is noteworthy that these negative tests represent $35.7 \%$ of all BFP tests during the four peak months, and $33.3 \%$ of all BFP tests during the other eight months of the year. It is therefore apparent that the seasonal incidence observed cannot be attributed to a sporadic increase in the finding by the RCBTS of apparent BFPs which were not later confirmed by the reference laboratory.

\section{PATTERN OF RESULTS (table II)}

\section{VDRL Negative}

Of the 284 VDRL tests performed by the RCBTS and found subsequently to be negative for syphilis, 99 were reported negative when all five tests, including a repeat VDRL, were run by the reference laboratory. These 99 tests (table II, category A) were found in a total of 80 donors. It is of interest to note that on 26 occasions a negative result was found on a donor who previously ( 5 occasions) or subsequently (21 occasions) during the year gave a further positive result to VDRL testing in the RCBTS laboratory. Of the 21 on whom further information was available for 1974, two gave a negative VDRL only on the second subsequent testing. The remaining 19 donors all had positive VDRLs on at least one subsequent test, while on nine occasions, other non-specific screening tests besides the VDRL were found to be positive. The implications of these findings for the transfusion service are discussed below. 


\begin{tabular}{|c|c|c|c|c|c|c|c|}
\hline Pattern & $V D R L$ & $W R$ & KOL & RPCFT & $F T A-A B S$ & Number ${ }^{1}$ & $\%$ \\
\hline A & - & - & - & - & - & $99(80)$ & 34.9 \\
\hline B & + & - & - & - & - & $135(113)^{2}$ & $47 \cdot 5$ \\
\hline C & + & + & - & - & - & $5(4)$ & 1.8 \\
\hline D & + & + & + & - & - & $5(3)$ & 1.8 \\
\hline $\mathbf{E}$ & + & - & + & - & - & 14 (12) & 4.9 \\
\hline $\mathbf{F}$ & + & - & - & + & - & $10(9)$ & 3.5 \\
\hline G & + & - & + & + & - & $8(6)$ & $2 \cdot 8$ \\
\hline H & - & - & + & - & - & $6(6)$ & $2 \cdot 1$ \\
\hline $\mathbf{J}$ & - & + & + & - & - & $2(2)$ & 0.7 \\
\hline
\end{tabular}

Table II IMVS investigation of RCBTS BFP VDRL

${ }^{1}$ The larger figure is the total number of tests; the lesser figure in parentheses is the number of individual donors tested. The percentages do not differ.

'Of the 135 tests in this category, 97 (from 83 donors) were recorded as 'weakly reactive' and 38 (from 30 donors) were recorded as 'reactive'.

\section{Positive Reagin Tests}

Almost half the positive VDRL tests performed by the RCBTS were confirmed by the reference laboratory as being positive for the VDRL test only (135). Of these, designated category B in table II, 97 were reported as weakly reactive and 38 as reactive.

The Wassermann reaction was positive with the VDRL on five occasions in four donors (category C) while all three reagin applications (VDRL, WR, and Kolmer) were positive on a further five studies done on three donors (category D).

The Kolmer (Kolmer cardiolipin Wassermann reaction) was found positive with the VDRL (category E) on 14 occasions in 12 donors.

Alone or in combination with other reagin tests, the VDRL was found positive by the reference laboratory in $62.3 \%$ of those in whom it was found positive by the referring laboratory.

Of particular interest were the eight donors in categories $\mathrm{H}$ and $\mathrm{J}$, in which the reference laboratory found the VDRL test negative but another reagin test positive. On six occasions this was the Kolmer alone, while on two other occasions the Kolmer and WR were both positive in the presence of a negative VDRL.

\section{Positive Reagin and Reiter Protein Tests}

On 18 occasions $(6.3 \%)$ in 15 donors, BFP reactions were observed in both the reagin and Reiter protein tests. On 10 occasions, the RPCFT was positive together with the VDRL (category $\mathrm{H}$ ), while on the remaining eight occasions the Kolmer was positive in addition to the VDRL and RPCFT. On no occasion was a positive RPCFT obtained without a positive reagin reaction.

\section{Repeated BFP Results}

Males and females are represented equally among the 36 donors found to have BFP results on more than one occasion during the year. Their average ages, 35.7 years for men and 38.5 years for women, differ somewhat from the overall figures above. The 26 donors who were found to have BFP results on two occasions have given between 2 and 74 previous donations, averaging $17.7 ; 10$ of them had given less than 10 donations. The seven donors who showed BFP reactions on three occasions had given between 15 and 39 donations, averaging 29 each, while the three donors, all women, showing BFP reactions on four occasions, had given 7,17 , and 60 donations respectively.

\section{Discussion}

\section{INFECTION AND BLOOD TRANSFUSION}

The possibility of transmitting an infective illness by blood transfusion is one which must be taken into account whenever a clinician weighs the possible risks of transfusion and sets these alongside the advantages he hopes to gain for his patient. The clinician relies on the transfusion service to exclude, either by history or laboratory test, or both, those donors whose blood may represent a potential infective hazard to a recipient. In some instances, such an assessment must be made on the basis of history alone, since no reliable screening test is available for the carrier state of malaria or cytomegalic virus infection.

For syphilis, and more recently hepatitis $\mathbf{B}$, routine screening tests are available and are mandatory before blood is transfused.

\section{SCREENING TESTS FOR SYPHILIS}

Two forms of tests are available for screening blood for the presence of syphilis-non-specific tests for the presence of reagin and specific tests for the presence of antitreponemal antibody.

Reagin is an immunoglobulin found in all human sera. In a number of clinical conditions, including syphilis, the amount present increases. At about the fourth and fifth week of primary syphilis infection, measurable amounts of reagin can be demonstrated 
in the serum. A colloidal suspension of animal tissue lipids will react with reagin present in serum by visible agglutination or by complement fixation. Of the several screening tests available, the VDRL is reliable, reproducible, and is probably the most widely used, since, as Garner and Backhouse (1971) point out, flocculation tests are preferred to complement fixation tests for routine screening purposes on the grounds of cost and technical complexity.

The specific tests for treponemal antibody are usually confined to reference laboratories. The Reiter protein antigen is obtained from disrupted washed Reiter treponemes and is not specific for $T$. pallidum. Occasional false positive reactions are seen when an antibody is present against the lipopolysaccharide antigens in protein extract. Schofield (1972) suggested that because a different mechanism operates in this test from that applying in the reagin test, the likelihood of coexistent biological false positives is small. However, in this series 18 sera gave biological false positive results to both reagin and Reiter protein tests (categories $F$ and $G$, table II). These represented $6.3 \%$ of all positive tests found at the RCBTS and $9.7 \%$ of all BFP tests confirmed by the reference laboratory.

INCIDENCE OF BFP REACTIONS FOR SYPHILIS Although screening tests for syphilis are performed by most laboratories, little information exists concerning the frequency of occurrence of BFP reactions to these tests within the 'normal' community. Although Garner (1970) found an incidence of BFP within her problem group of $6.2 \%$, routine screening of a much larger sample produced 50 BFP reactions from 21924 sera, an incidence of $0.23 \%$. As discussed below, although regular blood donation has been proposed as one of the causative factors in producing BFP reactions (Garner and Backhouse, 1970), the incidence of BFP tests for syphilis in the South Australian blood donation population of $0.39 \%$ does not differ significantly from the routine screening figure of Garner (1970). This percentage incidence for South Australia is reduced to $0 \cdot 25 \%$ if the IMVS-confirmed BFP results only are taken into account, and the tests found negative by the reference laboratory are omitted.

As there are less female donors on the panel than male donors, the finding of slightly more female donors with BFP results confirms previous statements that BFP results are commoner in females than males (Garner, 1970). In this present series, there is a somewhat larger number of young women than expected from previous evidence (Beal, 1965), and prolonged involvement with blood donation cannot be invoked as an explanation of the BFP result in at least some of these donors, since 46 of the 120 female donors are under 25 years of age and would have been involved with blood donation only since the age of 18 at the earliest.

Pregnancy is a recognized cause of BFP reactions for syphilis, but in theory, at least, this cannot explain these present findings since blood is not taken from donors known to be pregnant. In some other biological situations the pseudopregnant state induced by oral contraceptive ingestion mimics the pregnant state, and it may be that the occurrence of a BFP reaction for syphilis might need to be added to the ever-growing list of biological effects of oral contraceptives.

The marked seasonal incidence noted in this study was somewhat surprising and does not appear to have been reported previously. Upper respiratory tract infection is listed among the causes of an acute BFP reaction for syphilis, and the apparent predilection for the colder months of the year may in some measure be due to this. However, blood donors are discouraged from donation when they have upper respiratory tract infection and for two to three weeks afterwards.

\section{CAUSES OF VDRL BFP REACTIONS}

BFP reactions to VDRL testing may be regarded as acute or chronic, the distinguishing feature between the two being the persistence of the BFB result for more than six months. The causes of acute ${ }^{+}$ BFP reaction (Garner, 1970; Schofield, 1972) include infections such as viral pneumonia, measles, chicken pox, hepatitis, infectious mononucleosis, malaria, recent vaccination and/or immunization, and narcotic addiction. Chronic biological false positive reactions are seen in collagen diseases, including rheumatoid arthritis, systemic lupus erythematosus and polyarthritis nodosa, cirrhosis, thyrotoxicosis, psoriasis, leprosy, major allergies, and blood donation.

BFP REACTIONS AND BLOOD DONATION Garner and Backhouse $(1970,1971)$ have included repeated blood donation as a cause of chronic BFP results in tests for syphilis, suggesting that blood loss may lead to excessive reagin production in some susceptible individuals. Garner (1970) earlier found 22 BFP reactions in 88 blood donor sera referred for investigation, presumably because they had positive screening tests at the referring blood collection centre. In their subsequent series, Garner and Backhouse (1970) found that most of the $21.3 \%$ of blood donors who were confirmed as having a BFP reaction retained these BFP results when tested after six months.

Verso (1971) took issue with the deductions made by these authors from their data, pointing out that 
the size of the donor panel sampled and the period of time represented in the study had not been assessed and the results compared with the overall incidence of BFP reactions in the community. He suggested that blood donors were the only segment of the population on whom a mass survey of this kind had been done.

The findings of this present study tend to support the points made by Verso (1971) and present a somewhat different picture from the data of the study by Garner and Backhouse (1970). In this present survey, 24 donors had confirmatory evidence of specific treponemal infection, an incidence of $9.3 \%$, compared with $28.8 \%$ for this category in the Garner and Backhouse series. They confirmed $21.3 \%$ as BFP reactions, and found $49.9 \%$ non-reactive to all tests; the comparative figures for these two categories in this present study are 59.8 and $30.9 \%$ respectively.

The number of donors found to have BFP results for syphilis screening on more than one occasion during the year are too small to permit conclusions to be drawn. Certainly these donors tended, if female, to be older than the average, and the average number of donations given by those found positive on three and four occasions during the year was higher than by those found positive on two occasions only. However, these figures for average donations must be seen in the context of the transfusion service as a whole, in which the average number of donations given by presently active donors is 16.4 . Further follow-up of this aspect of the study seems indicated.

While it is recognized that the presence of chronic BFP reaction for syphilis may be the first indication of the presence of an underlying disease, among those disorders known to be associated with chronic BFP tests, there is no evidence from this present study to indicate that any of the donors in this category is suffering from any of these disorders.

\section{NEGATIVE CONFIRMATORY TESTS}

The reference laboratory at the Institute of Medical and Veterinary Science failed to confirm the positive VDRL result obtained by the Red Cross Blood Transfusion Service laboratory on 99 occasions in 80 donors. This percentage, less than in the series of Garner and Backhouse (1970), becomes smaller when it is remembered that at least $25 \%$ of these 80 donors gave positive VDRL reactions on other occasions before or after the test in question. There were also nine occasions on which the reference laboratory found negative results to repeat VDRL testing but positive results for other reagin tests.

An apparent discrepancy between the referring and reference laboratories must be viewed in the light of the rationale applying to VDRL screening in the referring laboratory. The VDRL test is used in the transfusion service as a screening test, performed in the interests of the safety and protection of the recipient. Therefore, if there exists any doubt at all about the VDRL test result being negative, the transfusion service will regard the VDRL test as being positive and refer the serum concerned for a complete workup by the reference laboratory. Such referrals account for one-third of the total, and this is a relatively consistent finding month by month throughout the year (fig 3).

While it may be possible to reduce the percentage of sera referred for investigation which are found subsequently to be negative for all tests, such a reduction should not be at the risk of the transfusion service retaining any doubt about the negativity of their single-barrelled screening procedure.

\section{References}

Beal, R. W. (1965). An age survey of South Australian blood donors. Med. J. Aust., 2, 519-524.

Beal, R. W. (1967). A prospective study of South Australian blood donors: 1. Static information. Med. J. Aust., 1, 749-755.

Garner, M. F. (1970). The biological false positive reaction to serological tests for syphilis. J. clin. Path., 23, 31-34.

Garner, M. F. and Backhouse, J. L. (1970). Chronic biological false-positive reactions to serological tests for syphilis in blood donors. J. clin. Path., 23, 478-480.

Garner, M. F. and Backhouse, J. L. (1971). Testing of blood donors for syphilis. Med. J. Aust., 1, 1374-6.

Keynes, G. (1949). Blood Transfusion, pp. 190 and 336. John Wright, Bristol.

Moore, J. E. and Mohr, C. F. (1952). Biologically false positive serologic tests for syphilis. J. Amer. med. Ass., 150, 467-473.

Schofield, C. B. S. (1972). Sexually Transmitted Disease, pp. 55-62. Churchill Livingstone, Edinburgh and London. Verso, M. L. (1971). Testing of blood donors for syphilis, Med. J. Aust., 2, 281. 